\title{
Anticorps biosimilaires versus princeps
}

\section{L'expérience}

en rhumatologie

et les biosimilaires du

trastuzumab en oncologie

Xavier Pivot ${ }^{1}$, Philippe Goupille ${ }^{2}$

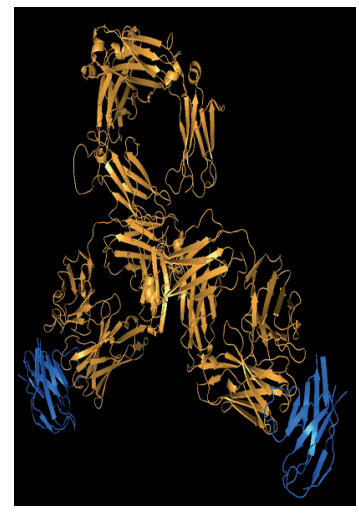

${ }^{1}$ Centre Paul Strauss, Institut de Cancérologie de Strasbourg, Strasbourg France.

${ }^{2} E A 7501$, GICC, Université de Tours ; Service de Rhumatologie,

CHRU de Tours, France.

philippe.goupille@univ-tours.fr

\section{Qu'est-ce qu'un biosimilaire?}

Un biosimilaire est une substance similaire à un médicament biologique de référence, dont le brevet est tombé dans le domaine public, et qui peut donc être « copié » [1]. Cette notion de similarité sous-entend qu'il y a des différences avec la molécule originale; en effet, un médicament biologique est une substance très complexe, produite à partir d'une cellule ou d'un organisme vivant, impliquant ainsi une certaine part de variabilité. La complexité de fabrication des anticorps thérapeutiques de par la biotechnologie que cette fabrication nécessite, fondée sur du vivant, ainsi que la complexité de la molécule à la fois du fait de sa taille (plus de 150000 Daltons) et de sa géométrie dans l'espace, rend illusoire l'obtention d'une parfaite copie de ces anticorps. Un biomédicament n'est donc jamais un composé parfaitement homogène, et il en sera de même pour ses biosimilaires. Dans ce contexte, les autorités ont développé le concept de «biosimilaire » correspondant à des molécules non parfaitement identiques mais qui remplissent exactement les mêmes fonctions biologiques [2-4]. Enfin, il faut noter que la plupart des biomédicaments princeps actuellement commercialisés ont fait l'objet de modifications, parfois majeures, de leurs procédés de production, modi- fications jugées suffisamment importantes pour être signalées aux chambres d'enregistrement des biomédicaments ; par exemple, pour l'infliximab (Remicade ${ }^{\circledR}$ ), 50 modifications ont été rapportées entre 1998 et 2016 (Figure 1) [5]. Si l'on est un peu provocateur, on peut ainsi poser la question suivante : le Remicade ${ }^{\circledR}$ de 2019 est-il un biosimilaire du Remicade ${ }^{\circledR}$ de 2000 ? En d'autres termes, n'y a-t-il pas plus de différences entre le Remicade ${ }^{\circledR}$ de 2019 et le Remicade ${ }^{\circledR}$ de 2000, qu'entre le Remicade ${ }^{\circledR}$ de 2019 et ses biosimilaires ?

Pour être validés comme biosimilaires, ces produits doivent répondre à un cahier des charges très strict (Tableau I) [2-4]. En effet, pour bénéficier d'une approbation de l'European Medicines Agency (EMA) et de la Food and Drug Administration (FDA), ces produits doivent démontrer qu'ils sont hautement biosimilaires au biomédicament princeps. Ainsi, ils doivent avoir la même formule biologique (même séquence en acides aminés), être produits par la même lignée cellulaire, même si les techniques de purification et les excipients peuvent différer. Le procédé global de fabrication doit être intégralement décrit et doit satisfaire aux critères de qualité d'un biomédicament. Enfin, contrairement aux génériques qui concernent des molécules chimiques, ils doivent démontrer, via des essais cliniques, un profil d'efficacité et de tolérance comparable au biomédicament princeps ; ces essais doivent comporter un essai de phase I d'équivalence pharmacocinétique et un essai de phase III d'efficacité clinique dans l'une des indications du biomédicament princeps (Figure 2).

Un biosimilaire ne doit pas être confondu avec un générique, dont l'acceptabilité est variable dans notre pays. En effet, un médicament 


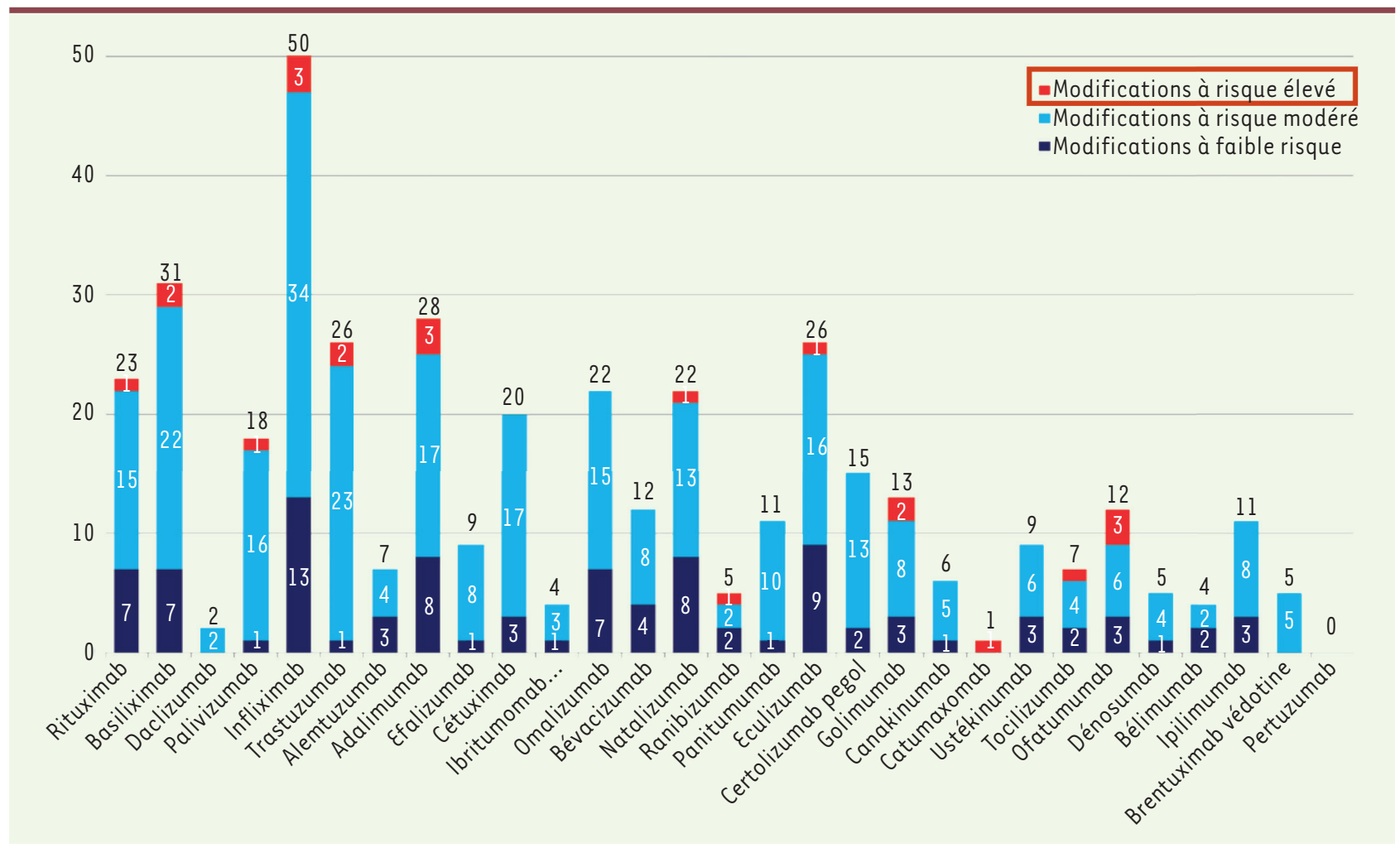

Figure 1. Modifications apportées aux biomédicaments au cours de leur développement.

générique est la copie d'un médicament chimique dont le brevet est tombé dans le domaine public ; il doit avoir strictement la même composition qualitative et quantitative en principes actifs, la même forme pharmaceutique et la même biodisponibilité, seul l'excipient pouvant varier. À la différence des biosimilaires, aucun essai clinique n'est requis pour valider son efficacité clinique, les 2 produits (princeps et générique) étant identiques.

\section{Spécificités des biosimilaires}

De nouvelles notions ont été développées avec l'avènement des biosimilaires :

L'extrapolation : compte tenu du coût des essais cliniques, et afin d'obtenir un moindre coût pour les biosimilaires, il est impossible d'exiger que leur développement reproduise l'intégralité de celui d'un princeps; ainsi, si un essai de phase III, dans une des indications du princeps, confirme la non-infériorité du biosimilaire, son efficacité peut alors être extrapolée à l'ensemble des indications du princeps. À titre d'exemple, un biosimilaire d'infliximab (anticorps chimérique anti-tumor necrosis factor-alpha, TNF- $\alpha$ ) ayant démontré son efficacité dans la polyarthrite rhumatoïde, a obtenu une autorisation de mise sur le marché (AMM) pour l'ensemble des indications du princeps, y compris pour le traitement des maladies inflammatoires chroniques de l'intestin ( $\mathrm{MICl}$ ), soulevant initialement une certaine réserve de la part des gastro-entérologues. Finalement, l'usage et le recul disponible sur l'utilisation du biosimilaire ont validé ce concept d'extrapolation.
La substitution : c'est l'acte par lequel le pharmacien délivre un produit autre que celui prescrit par le médecin ; ce principe, bien accepté pour les génériques, a fait l'objet de nombreux questionnements pour les biosimilaires : majoration du risque d'immunisation, traçabilité du produit pour le suivi de pharmacovigilance. À ce jour, et en l'absence de décret ad hoc, la substitution d'un princeps pour un biosimilaire ou entre biosimilaires, par un pharmacien, n'est pas autorisée.

L'interchangeabilité : elle permet de passer d'un princeps à son biosimilaire, d'un biosimilaire au princeps, ou d'un biosimilaire à un autre biosimilaire. Un rapport de l'agence nationale de sécurité du médicament et des produits de santé (ANSM) intégrant les données disponibles des registres norvégien et danois $[6,7]$ indique que «au vu de l'évolution des connaissances et de l'analyse continue des données d'efficacité et de sécurité des médicaments biosimilaires, une position excluant toute interchangeabilité en cours de traitement ne paraît plus justifiée » [8].

À ce jour, ni substitution, ni interchangeabilité ne sont autorisées pour le pharmacien.

\section{Pourquoi des biosimilaires?}

Les biomédicaments ont été une avancée majeure dans le traitement de nombreuses maladies chro- 


\begin{tabular}{ll}
\hline Caractéristiques & Exigences $\boldsymbol{\varepsilon}$ MA et FDA \\
\hline Séquence acides aminés & Pas de modification du moindre acide aminé \\
\hline Efficacité & Doit se rapprocher du produit référent \\
\hline Voie d'administration & La même que le produit référent \\
\hline Structure, modifications post-traductionnelles & $\begin{array}{l}\text { La plus proche du produit référent, avec des études démontrant que les différences } \\
\text { n'ont pas d'effet sur l'efficacité ou la tolérance }\end{array}$ \\
\hline Études cliniques & Exigences $\varepsilon$ MA et FDA \\
\hline Pharmacocinétique & $\begin{array}{l}\text { Etudes comparatives chez l'homme, une seule dose } \\
\text { Efficacité }\end{array}$ \\
\hline Tolérance & Au moins une étude d'équivalence \\
\hline Immunogénicité & Au moins une étude d'équivalence \\
\hline
\end{tabular}

Tableau I. Exigences de l'EMA et de la FDA pour le développement d'un biosimilaire.

niques, notamment en Rhumatologie. Toutefois, leur coût élevé soulève de nombreuses questions. Ainsi, l'étanercept (une protéine de fusion contenant l'ectodomaine du récepteur de type II du TNF- $\alpha$ fusionné à une région $\mathrm{Fc}_{c}$ d'IgGl humaine) et l'adalimumab (un $\mathrm{AcM}$ humain anti-TNF- $\alpha$ ) figurent, depuis plusieurs années, parmi les médicaments les plus onéreux pour l'Assurance maladie, I'infliximab faisant quant à lui partie des 3 médicaments hospitaliers les plus coûteux [9]. En 2014, selon l'Assurance Maladie, les dépenses annuelles de remboursement des anti-TNF- $\alpha$ s'élevaient à 280 millions d'euros pour l'infliximab (Remicade ${ }^{\circledR}$ ), 395 millions pour l'adalumumab (Humira ${ }^{\circledR}$ ) et 264 millions pour l'étanercept (Enbre|${ }^{\circledR}$ ) [10]. Les dépenses de biomédicaments et de thérapeutiques ciblées synthétiques ont ainsi atteint, pour les spécialités de Rhumatologie, Gastro-Entérologie et Dermatologie, un total de 1,5 milliard d'euros en 2017 (Tableau II). Ainsi, pour les deux AcM anti-TNF- $\alpha$ utilisés en ville $\left(\left.\varepsilon n b r e\right|^{\circledR}\right.$ et Humira $\left.{ }^{\circledR}\right)$, une substitution de $40 \%$ représenterait une économie de près de 58 millions d'euros, expliquant ainsi que le projet de loi de financement de la Sécurité sociale 2018 (PLFSS 2018) fixe un objectif de 40 millions d'euros d'économies grâce à une utilisation large des biosimilaires.

\section{L’expérience en rhumatologie}

Les premiers biosimilaires mis à disposition en rhumatologie ont été ceux de l'infliximab (médicament hospitalier administré par voie intraveineuse, princeps : Remicade ${ }^{\circledR}$ ), le CT-P13 (Inflectra ${ }^{\circledR}$ et Remsima ${ }^{\circledR}$ ) en 2015, puis le SB2 (Flixabi®) en 2017. Concernant le CT-P13, tant les études réalisées dans le cadre du plan de développement (PLANETAS, phase I dans la spondylarthrite, et PLANETRA, phase III dans la polyarthrite rhumatoïde) [11, 12], que les données de la vraie vie [6, 7] ont montré une équivalence en termes d'efficacité, de tolérance et d'immunogénicité comparativement au princeps, avec cependant un taux de retour au princeps d'infliximab de 10 à $20 \%$ chez les patients
$[13,14]$. Les rhumatologues disposent maintenant de biosimilaires du rituximab (princeps, Mabther ${ }^{\circledR}$ ), mais également d'AcM anti-TNF administrés par voie souscutanée, deux pour l'étanercept (princeps, Enbrel ${ }^{\circledR}$ ) et quatre pour l'adalimumab (princeps, Humira ${ }^{\circledR}$, biomédicament au chiffre d'affaire le plus élevé, toutes spécialités confondues, de l'ordre de 18 milliards d'euros en 2018), dont le taux de prescription augmente progressivement, mais avec une cinétique inférieure à celle d'autres pays européens.

\section{Quelles incitations et quel impact financier? Quelle politique de prix du Comité économique des produits de santé (CEPS) sur les médicaments biosimilaires?}

- Médicaments hospitaliers (liste en sus, ex : rituximab/ infliximab) : à l'arrivée du premier biosimilaire du biologique de référence, baisse du tarif de responsabilité (TR) de $30 \%$, même TR pour le produit de référence et ses biosimilaires, prix nets négociés par les acheteurs/ pharmaciens. La différence entre TR et prix net, appelée $\varepsilon M I$ (écart médicament indemnisable), est répartie à $50 / 50$ entre l'assurance maladie et la direction financière de l'établissement.

À titre d'exemple, ce dispositif, fondé sur la substitution de l'infliximab princeps par le biosimilaire, mais également les réductions du coût de l'infliximab princeps, ont permis de réduire considérablement le coût du traitement par l'infliximab au CHU de Tours (Tableau III) ; ainsi, en comparant les années 2015 et 2018, avec un même nombre de patients traités par l'infliximab en Rhumatologie et Gastro-Entérologie, le coût 


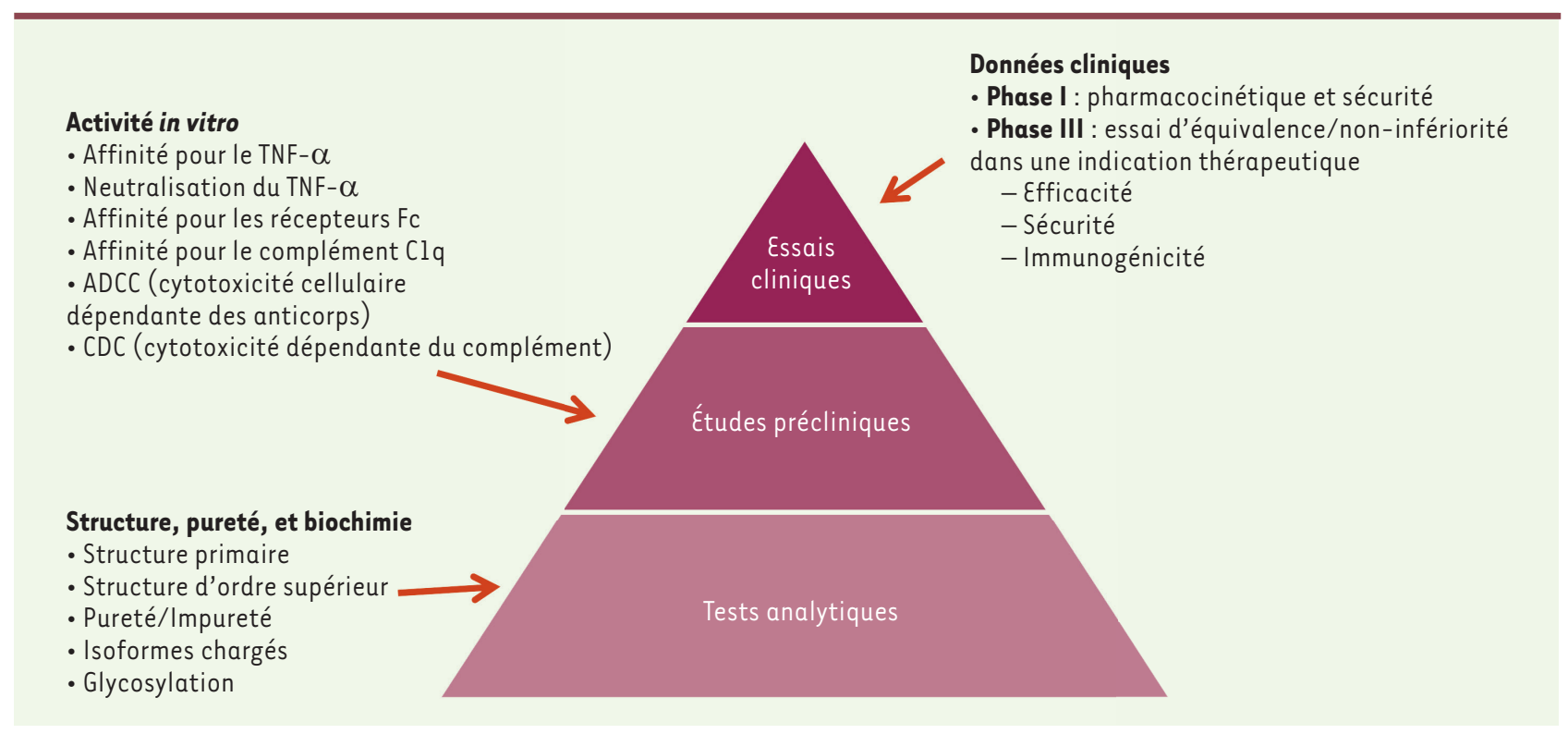

Figure 2. Développement d'un biomédicament biosimilaire.

du traitement est passé de 4 millions d'euros à 1,3 million d'euros, soit une réduction de 2,7 millions d'euros (Tableau III).

- Médicaments délivrés en ville (ex : étanercept/adalimumab) : à l'arrivée du premier biosimilaire, baisse de prix du produit princeps de $20 \%$, prix des biosimilaires inférieur de $40 \%$ par rapport au prix initial du produit princeps, baisses programmées à $18 / 24$ mois en fonction de la part de marché des biosimilaires. Dans le cadre de l'article 51 de la Loi de Santé, deux circulaires ont été publiées successivement en 2018 et 2019 , concernant notamment l'incitation à la prescription des biosimilaires de l'étanercept et de l'adalimumab [15, 16]. Ces circulaires concernent deux dispositifs d'intéressement qui s'excluent : le premier permet systématiquement un retour vers l'établissement prescripteur de biosimilaire de $20 \%$ de la différence de coût entre le princeps et le biosimilaire; le second, pour lequel à chaque fois une quarantaine d'établissements ont été sélectionnés en France, élève ce taux de retour à $30 \%$, vers le service prescripteur. À titre d'exemple, au CHU de Tours ce « dispositif à $30 \%$ » a permis de financer du temps médical en Rhumatologie et Gastro-Entérologie, du temps Pharmacien, et du temps pour infirmières d'éducation thérapeutique, financements qui seront bien sûr validés par l'obtention des taux de pénétration de biosimilaires que nous avons indiqués dans le dossier remis à l'ARS (Agence régionale de santé). Les premiers retours, notamment concernant la circulaire de 2018 sur les biosimilaires d'étanercept, sont encourageants quant à la cinétique du taux de pénétration des biosimilaires, et laissent espérer des gains financiers majeurs permettant de financer d'autres innovations et/ou de traiter un plus grand nombre de patients avec la même enveloppe budgétaire.

\section{Les biosimilaires du trastuzumab en oncologie}

Le trastuzumab (Herceptine ${ }^{\circledR}$ ) est une molécule qui a marqué l'histoire des traitements du cancer du sein, non seulement en raison de son efficacité au stade métastatique avec une prolongation de la survie, mais surtout du fait de l'amplitude du bénéfice observé dans le traitement néo-adjuvant des cancers du sein HER2 (human epidermal growth factor receptor factor 2)-positif [17]. L'expiration du brevet en 2017 en Europe a permis la copie de cette molécule [18]. Quatre biosimilaires du trastuzumab ont obtenu un enregistrement par l'EMA et plusieurs sont en cours de développement et obtiendront probablement un enregistrement dans les mois à venir.

\section{Équivalence biologique}

Le développement du biosimilaire doit démontrer la parfaite équivalence à la molécule originale au niveau de la séquence en acides aminées, en termes de glycosylations, de structure tridimensionnelle, de capacité de liaison à l'antigène cible, d'impact sur les voies de signalisation après fixation, d'induction éventuelle de CDC ou d'ADCC. De même, l'activité anti-proliférative et anti-tumorale, lorsqu'il s'agit d'anticorps comme le trastuzumab, sur les cellules de lignées cellulaires et dans des modèles de xénogreffes chez l'animal doit être parfaitement équivalente à la molécule de référence. En général, les résultats de cette évaluation biologique sont annexés en données supplémentaires dans les publications cliniques sauf pour le PF-05280014 qui a référencé un article complet sur ses résultats [19-21].

\section{Étude pharmacocinétique chez l'homme}

L'étude de l'équivalence pharmacocinétique a été réalisée pour tous les biosimilaires du trastuzumab chez des hommes indemnes comme cela est recommandé 
Dépenses Assurance maladie Ville 2016-2017 (source Medic’AM)

\begin{tabular}{|c|c|c|c|c|}
\hline PRODUITS & $\begin{array}{c}\text { Montant remboursé } \\
\text { Année } 2016\end{array}$ & $\begin{array}{c}\text { Montant remboursé } \\
\text { Année } 2017\end{array}$ & Croissance $€$ & Croissance \% \\
\hline TOTAI ENBREL & 237469903 & 220384652 & $-17085251 €$ & $-7 \%$ \\
\hline TOTAL BIOSIMILAIRES Etanercept & & 8711713 & $8711713 €$ & \\
\hline TOTAL Etanercept & 237732020 & 229096365 & $-8635655 €$ & $-4 \%$ \\
\hline Total HUMIRA & 465245755 & 482762416 & $17516662 €$ & $4 \%$ \\
\hline TOTAL CIMZIA & 42317877 & 47106412 & $4788536 €$ & $11 \%$ \\
\hline TOTAL ANTI-TNF SC & 839203962 & 854902347 & $15698385 €$ & $2 \%$ \\
\hline ORENCIA + ROACTEMRA SC & 60462316 & 72398341 & $11936025 €$ & $20 \%$ \\
\hline TALZ+0TEZLA & 1131050 & 17063325 & $15932275 €$ & $1409 \%$ \\
\hline TOTAL COSENTYX & 12290935 & 61244902 & $48953967 €$ & $398 \%$ \\
\hline TOTAL STELARA & 62139867 & 77453155 & $15313289 €$ & $25 \%$ \\
\hline TOTAL JAK Inh & & 630421 & $630421 €$ & \\
\hline TOTAL DÉPENSES AMBULATOIRES & 975228130 & 1083692491 & $108464361 €$ & $11 \%$ \\
\hline
\end{tabular}

Dépenses hospitalières de médicaments 2016-2017 valorisés TR (source GERS)

\begin{tabular}{|c|c|c|c|c|}
\hline PRODUITS & $\begin{array}{c}\text { Montant remboursé } \\
\text { Année } 2016\end{array}$ & $\begin{array}{c}\text { Montant remboursé } \\
\text { Année } 2017\end{array}$ & Croissance $€$ & Croissance \% \\
\hline REMICADE & 271931923 & 205147922 & -66784001 & $-25 \%$ \\
\hline Biosimilaires infliximab & 75691461 & 129827029 & 54135568 & $72 \%$ \\
\hline Total infliximab (TR) & 347623384 & 334974951 & -12648433 & $-4 \%$ \\
\hline Infliximab Estimé prix net & 250000000 & 200000000 & -50000000 & $-20 \%$ \\
\hline Anti-TNF SC & 7684852 & 7265455 & -419397 & $-5 \%$ \\
\hline ORENCIA IV+ ROACTEMRA IV & 61965108 & 62926447 & 961339 & $2 \%$ \\
\hline MABTHERA $10 \%$ & 30904515 & 30104864 & -799651 & $-3 \%$ \\
\hline TOTAL HÔPITAL (prix TR) & 448177859 & 435271717 & -12906142 & $-3 \%$ \\
\hline
\end{tabular}

Tableau II. Dépenses de médicaments biologiques et pour les « petites » molécules: maladies inflammatoires en rhumatologie/gastro-entérologie/dermatologie en 2017 (données de l'assurance maladie). ENBREL (étanercept, protéine de fusion anti-TNF) ; CIMZIA (certolizumab pegol) (anti-TNF- $\alpha$ ); COSENTYX (sécukinumab) (anti-IL-17A); JAK inh (inhibiteurs de Janus Kinase) ; MABTHERA (rituximab) (anti-CD20); ORENCIA (abatacept) (anti-CTLA4) ; REMICADE (infiximab) (anti-TNF- $\alpha$ ); OTEZLA (aprémilast) (inhibiteur de la phosphodiestérase 4 (PDE4)) ; ROACTEMRA (tolicizumab) (anti-IL-6), SIMPONI (golimumab) (anti-TNF- $\alpha$ ) ; STELARA (ustékinumab) (anti-IL-12/-23) ; TALTZ (ixékizumab) (anti-IL-17A)

par les agences d'enregistrement. L'objectif est d'éviter d'exposer des femmes qui sont plus à risque de développer un cancer du sein HER2positif. En cas de développement d'anticorps anti-trastuzumab, cela pourrait être en effet péjoratif pour l'optimisation de leur traitement. Ces phases I chez l'homme ont consisté en une injection avec une équivalence basée sur le critère d'AUC et/ou de concentration maximale $\left(C_{\max }\right)$ en comparant le biosimilaire au trastuzumab produit aux
États-Unis et/ou en Europe [20-27]. Tous les autres paramètres pharmacocinétiques sont aussi évalués (clairance, demi-vie). Au total, tous les biosimilaires testés ont démontré leur équivalence avec parfois, de manière anecdotique, une différence minime entre I'Herceptine ${ }^{\circledR}$ d'origine américaine et européenne par rapport au biosimilaire $[20,23,26]$. 


\begin{tabular}{|ccc|}
\hline Année & Coût en $€$ & Nombre de patients traités \\
\hline 2015 & 4 millions & 353 \\
\hline 2016 & 3,5 millions & 341 \\
\hline 2017 & 2,5 millions & 351 \\
\hline 2018 & 1,3 million & 358 \\
\hline $2015-2018$ & $-2,7$ millions & +5 \\
\hline
\end{tabular}

Tableau III. Évolution du coût de l'infliximab (rhumatologie et gastroentérologie) au CHU de Tours entre 2015 et 2018.

Ces études pharmacocinétiques avec une administration unique chez l'homme sain ne sont probablement pas suffisantes pour démontrer l'équivalence. En effet, nous savons que la pharmacocinétique du trastuzumab se stabilise après des administrations itératives au-delà du $5^{\text {e }}$ cycle, de même que l'exposition de patientes avec une tumeur HER2-positif pourrait avoir un impact sur la pharmacocinétique [28, 29]. Par ailleurs, le paramètre clé dans l'activité du trastuzumab est la concentration biologique minimale efficace fixée à $20 \mu \mathrm{g} / \mathrm{L}$, la concentration devant toujours être au-dessus de cette valeur seuil pour maintenir l'activité biologique. Cette concentration vallée a été estimée ou parfois non mesurée dans les phases I ; de ce fait, une évaluation pharmacocinétique dans une étude ancillaire lors des études randomisées mesurant l'activité clinique est souvent venue compléter la démonstration d'équivalence pharmacocinétique [30-32]. Pour les anticorps comme AB980, SB3 et CPT6, la démonstration de l'équivalence en termes de concentration vallée au $21^{\mathrm{e}}$ jour a été apportée lors des grands essais de phase III. Seul le PF-05280014 a fait l'objet d'une étude de phase III propre, étude de pharmacocinétique qui a été réalisée à la fois pour compléter la phase I mais également pour aider la communication et l'enregistrement de ce biosimilaire avec des données d'une étude néo-adjuvante [33].

\section{Étude d'équivalence en termes d'activité clinique}

La deuxième grande partie du développement clinique d'un biosimilaire est fondée sur des études randomisées pour démontrer une activité équivalente entre la molécule de référence et le biosimilaire dans une population représentative de l'indication de la thérapie. Les paramètres clés pour évaluer ces études cliniques sont la définition du critère d'équivalence pour l'activité et les critères de démonstration statistique d'équivalence.

Le critère d'efficacité ne saurait reposer sur des objectifs de survie qui, pour des études d'équivalence, demandent de très nombreux évènements et un long suivi, ce qui va contre le concept du développement rapide à coût réduit de biosimilaires. La réponse objective à la phase métastatique peut être considérée par certains comme un critère d'évaluation même si, dans le cancer du sein HहR2-positif, certains sont plus réservés. En effet, jamais une molécule n'a été enregistrée sur le critère de réponse objective, jamais les réponses objectives n'ont été reliées à des survies [34]. Dans le cancer du sein, il existe un autre critère précoce après un traitement néo-adjuvant, l'obtention de la réponse complète histologique (pCR) [35]. Ce critère est relié à la survie et il a permis l'enregistrement de plusieurs molécules comme le pertuzumab ou du trastuzumab injecté par voie sous-cutanée. II est clair que le critère de réponse complète histologique est un critère simple et rigoureux : existence ou absence de tumeur. L'évaluation lors du traitement néo-adjuvant présente moins de facteurs de biais que l'évaluation de patientes présentant une tumeur HER2-positive métastatique (phase métastatique) : les patientes sont alors indemnes de tout traitement et il existe une variabilité moindre dans le bilan d'extension de la maladie. De ce fait, il semble licite de privilégier les traitements néo-adjuvants pour évaluer les nouveaux traitements anti-HER2, de même que pour démontrer l'équivalence clinique entre l'Herceptine ${ }^{\circledR}$ et les biosimilaires du trastuzumab [36].

Le deuxième paramètre clé est la démonstration statistique. L'équivalence est fondée sur des marges variables, entre 12 et $20 \%$, des intervalles de confiance variables, entre 90-95\%. Théoriquement, les marges d'équivalence sont déterminées par la volonté de préserver entre 60 et $50 \%$ de l'activité de l'Herceptine ${ }^{\circledR}$, estimée par la méta-analyse des études évaluant ce traitement dans l'indication étudiée. Au total, l'inclusion variable de certaines études, l'utilisation de la préservation de l'activité minimale correspondant à la borne inférieure de l'intervalle de confiance ou de la valeur médiane estimée, aboutit à des marges d'équivalence légèrement différentes. De plus, ces équivalences peuvent être fondées sur la différence absolue entre les taux de réponse complète ou sur les ratios réponses complète / réponse partielle.

\section{Résultats des principaux candidats}

Au total, le développement de trois biosimilaires a été fondé sur une étude clinique néo-adjuvante et celui de deux sur des études menées à la phase métastatique (Tableau IV). La première analyse d'une phase III démontrant l'équivalence entre un biosimilaire du trastuzumab et la molécule de référence a été publiée en 2017 par Rugo et al. [37]. La démonstration a été établie à la phase métastatique sur le critère de réponse objective. Pour des raisons liées à l'homogénéité de la qualité de la production, cette molécule n'est pas disponible à ce jour en Europe. Une grande compagnie a, quant à elle, démontré l'équivalence à la phase métastatique du PF-05280014 avec l'Herceptine ${ }^{\circledR}[38]$, mais une deuxième étude dédiée à la pharmacocinétique a été conduite en phase néoadjuvante avec l'obtention de taux de pCR similaires à 


\begin{tabular}{|c|c|c|c|c|c|}
\hline & ABP980 [17] & $\begin{array}{l}\text { Bioepis/Merck } \\
\text { SB3 [19] }\end{array}$ & CT-P6 [18] & $\begin{array}{l}\text { PF-05280014 } \\
{[20,27]}\end{array}$ & $\begin{array}{l}\text { MYL- } 14010 \\
{[26]}\end{array}$ \\
\hline Néo-adjuvant & $\checkmark$ & $\checkmark$ & $\checkmark$ & $\checkmark$ & \\
\hline N & 725 & 875 & 549 & 226 & \\
\hline Métastastique & - & - & $\ddot{\mathrm{u}}$ & $\ddot{u}$ & $\ddot{u}$ \\
\hline N & - & - & 475 & 707 & 458 \\
\hline Objectif principal & Total $p C R$ & Sein $p C R$ & $\begin{array}{c}\text { Néo-adj: total pCR } \\
\text { Méta: ORR }\end{array}$ & $\begin{array}{l}\text { Néo-adj: PK } \\
\text { Méta: ORR }\end{array}$ & ORR \\
\hline $\begin{array}{l}\text { Bornes Equivalences } \\
\text { (différence absolue) }\end{array}$ & $90 \%$ IC $\pm 13 \%$ & $95 \%$ IC $\pm 13 \%$ & $\begin{array}{c}\text { Néo-adj: } 95 \% \text { IC } \pm 15 \% \\
\text { Méta: } 95 \% \mathrm{IC} \pm 15 \%\end{array}$ & $\begin{array}{c}\text { Néo-adj: } 95 \% \\
\text { IC } 0,8-1,25 \\
\text { (risk ratio) }\end{array}$ & $95 \% \mathrm{IC} \pm 15 \%$ \\
\hline
\end{tabular}

Tableau IV. Résultats des principales études cliniques des candidats biosimilaires du trastuzumab. IC: intervalle de confiance ; ORR: objective response rate; pCR: pathological complete response ; PK: pharmacocinétique.

ceux attendus et à ceux observés dans les études concurrentes [33]. Un autre biosimilaire, le CPT-6, a vu son développement fondé sur une étude néo-adjuvante [31]. Cette étude sur le CPT-6 a été la première publiée et a confirmé l'équivalence, avec des marges de $15 \%$ en termes de réponse absolue, et en examinant la réponse complète histologique totale. Une AMM européenne a été obtenue en 2018 pour le CPT-6. Une étude néo-adjuvante a également été réalisée pour l'ABP980; l'équivalence a également été démontrée, même si la marge supérieure de l'intervalle de confiance de $13 \%$ est légèrement dépassée dans l'étude [30]. La relecture centralisée de la réponse complète histologique qui était prévue et réalisée de manière indépendante a retrouvé une différence de l'intervalle de confiance parfaitement incluse dans les marges d'équivalence. L'enregistrement de l'ABP980 en Europe a été obtenu en 2018. Enfin, un autre biosimilaire, le SB3, a été développé en néo-adjuvant, en regardant uniquement la réponse complète histologique dans la tumeur primitive du sein sans tenir compte des ganglions [32]. Cette nuance permet une évaluation plus stricte de l'activité du biosimilaire par rapport à la molécule originelle, mais le critère de réponse complète de la tumeur du sein est dans une proportion moindre reliée à la survie par rapport à la réponse complète histologique qui inclut le ganglion [39]. L'avantage est qu'il n'y a pas de biais par rapport au statut ganglionnaire qui est inconnu au début du traitement. Dans cette étude, la marge supérieure de l'intervalle de confiance était au-delà de la marge supérieure de la marge d'équivalence ( $17 \%$ versus $13 \%$ ). Ce résultat n'a pas bloqué la reconnaissance européenne, avec l'enregistrement de cet anticorps biosimilaire qui a été le premier approuvé, à l'automne 2017. La différence avec un intervalle de confiance qui est au-delà de la borne d'équivalence est expliquée par l'utilisation dans le bras Herceptine ${ }^{\circledR}$ d'une proportion de molécules présentant une modification de glycosylation, caractérisée par un taux moindre de fucose et de mannose. Ce déficit a été monitoré au cours du temps par le promoteur de l'étude avec une démonstration biologique de la moindre efficacité en terme non pas d'activité anti-proliférative mais d'ADCC, induit par ce déficit [40]. Cette hétérogénéité de qualité d’Herceptine ${ }^{\circledR}$, molécule de référence dans le bras contrôle, explique le résultat; au vu de l'ensemble de l'exercice de comparaison incluant la biologie, la pharmacocinétique et l'étude clinique d'activité, le SB3 a été enregistré.

$\varepsilon n$ conclusion, il y a donc trois biosimilaires enregistrés à la suite d'une étude clinique d'activité évaluée en néo-adjuvant (SB3, CPT-6, ABP980) et un (PF05280014) enregistré sur la base d'une étude menée à la phase métastatique (Tableau IV). D'autres acteurs sont aujourd'hui impliqués, I'un d'entre eux travaillant sur l'amélioration de son outil de production pour obtenir un accès au marché européen. Une hiérarchie en termes de confiance concernant la qualité des biosimilaires peut-elle être proposée ? La robustesse et la validité des études cliniques sont probablement un critère [41]. Privilégier, en termes de confiance, les études cliniques validées en néo-adjuvant pour une molécule aussi emblématique que l'Herceptine ${ }^{\circledR}$ pourrait apparaître raisonnable.

\section{Extrapolation des indications}

L'obtention du label de biosimilaire du trastuzumab autorise l'utilisation pour toutes les indications d'Herceptine ${ }^{\circledR}$, soit le cancer du sein métastatique en association avec le pertuzumab, en association avec le lapatinib, en phase néo-adjuvante et adjuvante, soit dans les cancers gastriques. 


\section{Impact économique}

Ce principe du biosimilaire est d'importance puisque, au-delà de la décroissance du prix du trastuzumab (2 baisses successives de tarifs de $15 \%$ chacune), les prix des biosimilaires ont réduit de $50 \%$ à $60 \%$ le coût par rapport au tarif national des premières offres à l'automne 2018.

\section{Conclusion}

En conclusion, « l'aventure biosimilaires » est fondée sur une optimisation de l'outil de production, c'est-à-dire les lignées cellulaires, leur résistance et leur capacité de production. L'enjeu est avant tout financier et sociétal. Cette optimisation va permettre un développement d'anticorps nettement moins onéreux et probablement de nouvelles thérapeutiques à des prix plus abordables. L'émergence de ces biosimilaires, maintenant fortement stimulée par les autorités de tutelle, permettra de faire des économies substantielles et de financer l'accès à d'autres molécules innovantes. $\diamond$

\section{SUMMARY}

Biosimilars antibodies: positioning compared to originators - the experience in rheumatology and the biosimilars of trastuzumab in oncology

Biosimilars have demonstrated their equivalence with biologic originators, according to rigorous specifications imposed by the regulatory agencies, the FDA and the EMA. Their development is justified by the very high cost of biopharmaceuticals, and strong incentives for their prescription lead us to hope substantial savings, allowing to finance other innovative molecules. Trastuzumab marked history of the treatment of breast cancer. Four biosimilars of trastuzumab are available for routine use and we will detail the key points of their development. $\diamond$

\section{LIENS D'INTÉRÊT}

Les auteurs déclarent n'avoir aucun lien d'intérêt concernant les données publiées dans cet article.

\section{RÉFÉRENCES}

1. Guidelines on evaluation of similar biotherapeutic products 5 SBPs, 2010 ; p. 1-34.

2. European Medecines Agency cfMpfHU. Guideline on similar biological medecinal products containing monoclonal antibodies - non clinical and clinical issues. http://wwwemaeuropaeu/ docs/en_GB/document_library/Scientif_guideline/2012/06/WC500128686pdf. 2012.

3. Agency EM. Guideline on similar biological products containing biotechnology-derived proteins as active sustance: non-clinical and clinical issues. http://wwwemaeuropaeu/docs/en_GB/ document_library/Scientif_guideline/2015/01/WC500180219pdf.2015.

4. Agencies UEM. Scientific considerations in demonstrating biosimilarity to a reference product, Guidance for Industry. http://wwwfdagov/downloads/drugs/ guidanceComplianceRegulatorylnformation/Guidances/UCM291128pdf. 2015.

5. Vezér B, Buzás Z, Sebeszta M, Zrubka Z. Authorized manufacturing changes for therapeutic monoclonal antibodies (mAbs) in European Public Assessment Report (EPAR) documents. Curr Med Res Opin 2016; $32: 829-34$.

6. Jorgensen KK, Olsen IC, Goll GL, et al. Switching from originatoir infliximab to biosimilar CT-P13 compared with maintained treatment with originator infliximab [NOR-SWITCH] : a 52-week, randomised, double-blind, non-inferiority trial. Lancet 2017 ; 389 : 2304-16.

7. Glintborg B, Sørensen IJ, Loft AG, et al. A nationwide non-medical switch from originator infliximab to biosimilar CT-P13 in 802 patients with inflammatory arthritis: 1-year clinical outcomes from the DANBI0 registry. Ann Rheum Dis 2017 ; 76 : 1426-31.
8. Agence Nationale de sécurité du médicament et des produits de santé. État des lieux sur les médicaments biosimilaires. Paris : Ansm, $2016: 1-28$.

9. ANSM. Analyse des ventes de médicaments en France en $2013 ; 2014$; p. 1-36 [http://www.ansm.sante.fr/content/download/64305/824219/ version/2/file/ANSM.Analyse-Ventes-Medicaments.2013.pdf].

10. Ghersinick-09072. In : Dépenses de médicaments de l'année 2014 et du premier semestre 2015. Paris : Caisse nationale d'assurance maladie, 2015 1-24.

11. Yoo DH, Prodanovic N, Jaworski J, et al. Efficacy and safety of CT-P13 (biosimilar infliximab) in patients with rheumatoid arthritis: comparison between switching from reference infliximab to CT-P13 and continuing CT-P13 in the PLANETRA extension study. Ann Rheum Dis $2017 ; 76: 355-63$.

12. Park $W, Y_{0 o} D H$, Miranda $P$, et al. Efficacy and safety of switching from reference infliximab to CT-P13 compared with maintenance of CT-P13 in ankylosing spondylitis: 102-week data from the PLANETAS extension study. Ann Rheum Dis 2017 ; 76 : 346-54.

13. Avouac J, Moltó A, Abitbol V, et al. Systematic switch from innovator infliximab to biosimilar infliximab in inflammatory chronic diseases in daily clinical practice: The experience of Cochin University Hospital, Paris, France. Semin Arthritis Rheum 2018 ; $47: 741-8$.

14. Scherlinger M, Germain V, Labadie C, et al. FHU ACRONIM. Switching from originator infliximab to biosimilar CT-P13 in real-life: the weight of patient acceptance. Joint Bone Spine 2018 ; 85 : 561-7.

15. Arrêté du 3 août 2018 relatif à l'expérimentation pour l'incitation à la prescription hospitalière de médicaments biologiques similaires délivrés en ville. Journal Officiel 17 août 2018.

16. Arrêté du 12 février 2019 relatif à l'expérimentation pour l'incitation à la prescription hospitalière de médicaments biologiques similaires délivrés en ville. Journal Officiel 15 février 2019.

17. Hortobagyi GN. Trastuzumab in the treatment of breast cancer. $N$ Engl J Med $2005 ; 353: 1734-6$.

18. Pivot X, Aulagner G, Blay JY, et al. Challenges in the implementation of trastuzumab biosimilars: an expert panel's recommendations. Anticancer Drugs $2015 ; 26: 1009-16$.

19. Hurst S, Ryan AM, Ng CK, et al. Comparative nonclinical assessments of the proposed biosimilar PF-05280014 and trastuzumab (Herceptin $\left.{ }^{\circledR}\right)$. BioDrugs 2014 ; 28 : 451-9.

20. Pivot X, Curtit $\varepsilon$, Lee YJ, et al. A Randomized phase I pharmacokinetic study comparing biosimilar candidate SB3 and trastuzumab in healthy male subjects. Clin Ther 2016 ; 38 : 1665-73e3.

21. Pivot X, Deslypere JP, Park LS, Kim MJ, Lee W, Lee J. A randomized phase I study comparing the pharmacokinetics of HD201, a trastuzumab biosimilar, with European Union-sourced herceptin. Clin Ther 2018; 40 : 396-405 e4.

22. Esteva FJ, Stebbing J, Wood-Horrall RN, Winkle PJ, Lee SY, Lee SJ. A randomised trial comparing the pharmacokinetics and safety of the biosimilar CT-P6 with reference trastuzumab. Cancer Chemother Pharmacol $2018 ; 81: 505-14$.

23. Hanes V, Chow V, Zhang N, Markus R. A randomized, single-blind, single-dose study evaluating the pharmacokinetic equivalence of proposed biosimilar ABP 980 and trastuzumab in healthy male subjects. Cancer Chemother Pharmacol $2017 ; 79: 881-8$.

24. Morita J, Tanaka M, Nomoto M, et al. Pharmacokinetic bioequivalence, safety, and immunogenicity of DMB-3111, a trastuzumab biosimilar, and trastuzumab in healthy Japanese adult males: results of a randomized trial. BioDrugs $2016 ; 30: 17-25$.

25. Zhou $X, Y_{u}$ J, Wang $W$, et al. A phase I dose-escalation study of a biosimilar trastuzumab in Chinese metastasis breast cancer patients. Springerplus. $2015 ; 4: 803$.

26. Yin D, Barker KB, Li R, et al. A randomized phase 1 pharmacokinetic trial comparing the potential biosimilar PF-05280014 with trastuzumab in healthy volunteers (REFLECTIONS B327-01). BrJ Clin Pharmacol 2014 ; 78 : 1281-90.

27. Wisman LA, De Cock EP, Reijers JA, et al. A phase I dose-escalation and bioequivalence study of a trastuzumab biosimilar in healthy male volunteers. Clin Drug Investig 2014 ; 34 : 887-94.

28. Bruno R, Washington CB, Lu JF, Lieberman G, Banken L, Klein P. Population pharmacokinetics of trastuzumab in patients with HER2+ metastatic breast cancer. Cancer Chemother Pharmacol 2005 ; 56 : 361-9.

29. Leyland-Jones B, Gelmon K, Ayoub JP, et al. Pharmacokinetics, safety, and efficacy of trastuzumab administered every three weeks in combination with paclitaxel. J Clin Oncol $2003 ; 21$ : 3965-71. 


\section{RÉFÉRENCES}

30. von Minckwitz G, Colleoni M, Kolberg HC, et al. Efficacy and safety of ABP 980 compared with reference trastuzumab in women with HER2-positive early breast cancer (LILAC study): a randomised, double-blind, phase 3 trial. Lancet Oncol 2018 ; 19 : 987-98.

31. Stebbing J, Baranau Y, Baryash V, et al. CT-P6 compared with reference trastuzumab for HER2positive breast cancer: a randomised, double-blind, active-controlled, phase 3 equivalence trial. Lancet Oncol $2017 ; 18: 917-28$

32. Pivot X, Bondarenko I, Nowecki Z, et al. Phase III, randomized, double-blind study comparing the efficacy, safety, and Immunogenicity of SB3 (trastuzumab biosimilar) and reference trastuzumab in patients treated with neoadjuvant therapy for human epidermal growth factor receptor 2-positive early breast cancer. J Clin Oncol $2018 ; 36$ : 968-74.

33. Lammer P, Dank M, Masetti R, et al. Neoadjuvant PF-05280014 (a potential trastuzumab biosimilar) versus trastuzumab for operable HER2+ breaast cancer. Br J Cancer 2018 ; 119 : 266-73

34. Pivot X, Thierry-Vuillemin A, Villanueva C, Bazan F. Response rates: a valuable signal of promising activity? Cancer J $2009 ; 15: 361-5$.

35. Cortazar P, Zhang L, Untch M, et al. Pathological complete response and long-term clinical benefit in breast cancer: the CTNeoBC pooled analysis. Lancet. 2014 ; 384 : 164-72.

36. Pivot X, Cox DG. A new era for treatment development in HER2-positive breast cancer. Lancet Oncol $2018 ; 19: 160-2$

37. Rugo HS, Barve A, Waller CF, et al. Effect of a proposed trastuzumab biosimilar compared with Trastuzumab on Overall Response Rate in Patients With ERBB2 (HER2)-Positive Metastatic Breast Cancer: A Randomized Clinical Trial. JAMA 2017 ; 317 : 37-47.
38. Pegram M, Tan-Chui $\varepsilon$, Freyman A, et al. A randomized, double blind study of PF-05280014 (a potential trastuuzmab biosimilar) vs trastuzumab, both in combination with paclitaxel, as first-line treatment for HER2-positive metastatic breast canecr. European Society for Medical Oncology (ESMO). 2017.

39. Pivot X, Bondarenko I, Nowecki Z, et al. A phase III study comparing SB3 (a proposed trastuzumab biosimilar) and trastuzumab reference product in HER2-positive early breast cancer treated with neoadjuvant-adjuvant treatment: final safety, immunogenicity and survival results. Eur J Cancer $2018 ; 93: 19-27$.

40. Kim S, Song J, Park S, et al. Drifts in ADCC-related quality attributes of Herceptin $(\mathrm{R})$ : Impact on development of a trastuzumab biosimilar. MAbs $2017 ; 9: 704-14$

41. Pivot $X$, Petit $\mathrm{T}$. Can we establish a hierarchy among trastuzumab biosimilar candidates? Br J Cancer $2018 ; 119$ : 263-5.

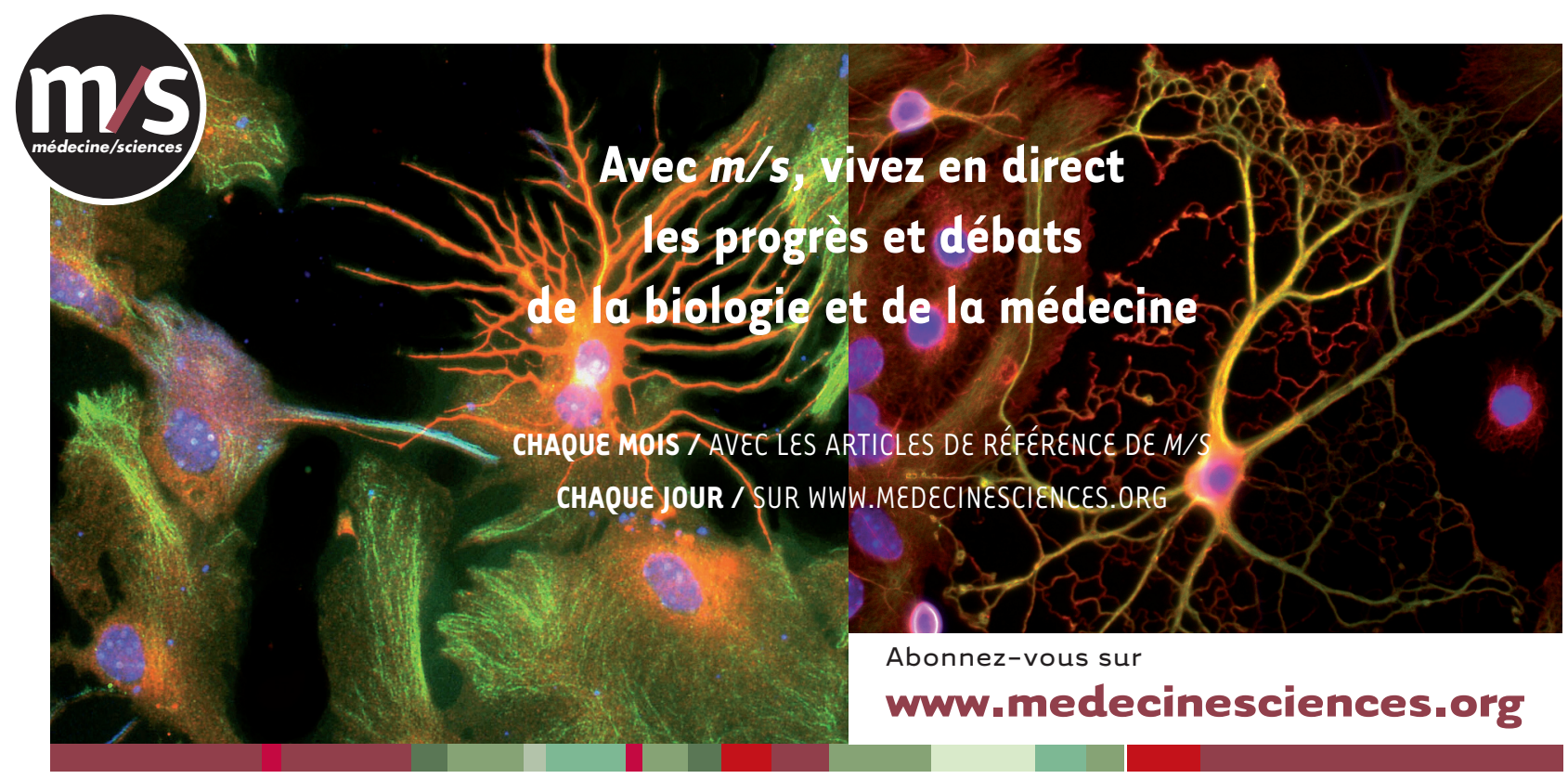

TIRÉS À PART

P. Goupille

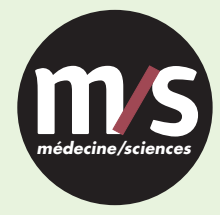

Tarifs d'abonnement $\mathrm{m} / \mathrm{s}-2019$

Abonnez-vous

à médecine/sciences
$>$ Grâce à $m / s$, vivez en direct les progrès des sciences biologiques et médicales

\section{Bulletin d'abonnement} page 1230 dans ce numéro de $\mathrm{m} / \mathrm{s}$

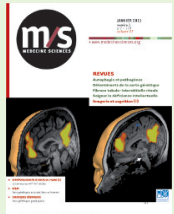

